\title{
Gastric giardiasis
}

C Doglioni, M De Boni, R Cielo, L Laurino, P Pelosio, P Braidotti, G Viale

\begin{abstract}
Aims: To assess the prevalence of gastric giardiasis in patients undergoing upper gastrointestinal endoscopy, and to define the clinicopathological correlates of gastric Giardia lamblia infection.

Methods: Consecutive gastric biopsy specimens $(n=15023)$ from 11085 patients, taken at Feltre City Hospital (north eastern Italy) from January 1986 to December 1991, were histologically and immunocytochemically examined for the occurrence of $G$ lamblia trophozoites. Three gastric biopsy specimens from patients harbouring $G$ lamblia infection, who repeated endoscopy before treatment, were also examined electron microscopically.
\end{abstract}

Results: Forty one patients $(0 \cdot 37 \%$ of the population study) harboured gastric giardiasis. All patients underwent upper gastrointestinal endoscopy because of dyspepsia, epigastric pain, or abdominal distension. Only two patients had diarrhoea at the time of investigation. Giardiasis was clinically unsuspected in all cases, although the nine patients who also had duodenal biopsies performed had concomitant intestinal giardiasis. Gastric giardiasis was invariably associated with chronic atrophic gastritis. Intestinal metaplasia of the gastric mucosa and Helicobacter pylori infection were found in 32 and 37 of the 41 patients with gastric giardiasis, respectively.

Conclusions: The invariable association of gastric giardiasis with chronic atrophic gastritis, most often showing intestinal metaplasia and $H$ pylori infection, indicates that a decreased gastric acidity is a prerequisite for localisation of $G$ lamblia to the gastric mucosa. Though its possible role as a gastric pathogen remains to be elucidated, these findings suggest that trophozoites should be carefully searched for when examining gastric biopsy specimens showing chronic atrophic gastritis.

\section{(F Clin Pathol 1992;45:964-967)}

Giardia lamblia was the first intestinal protozoa to be observed and described by Leeuwenhock in 1681, although it derives its name from Vilem Lambl, a Czech physician who identified it in faeces in 1859, and was formally considered its discoverer.

The pathogenicity of $G$ lamblia has been controversial for a long time, but it is now accepted that it causes disease in man. ${ }^{1}$ Infection is usually followed by an acute diarrhoeal illness, after an incubation period of 12 to 19 days; chronic giardiasis can also be accompanied by ill defined gastrointestinal symptoms. ${ }^{2}$ Diagnosis of infection relies on the identification of trophozoites in duodenal aspirates or cysts in stool. Immunological and genotypic assays have been introduced in clinical practice over the past few years. ${ }^{34}$ Histological examination of duodenal and jejunal biopsy specimens from patients with a clinical history of malabsorption permits direct visualisation of the trophozoites.

In infected patients $G$ lamblia characteristically localises in the proximal small intestine, but localisation in extraintestinal sites, including gall bladder, ${ }^{5}$ has also been reported. However, no mention is made that the organism could possibly be identified in gastric biopsy specimens.

Following the first observation of G lamblia trophozoites in a gastric biopsy specimen, we undertook a prospective and retrospective analysis for the presence of the organism in routine gastric biopsy specimens taken at the endoscopic unit, Feltre City Hospital.

\section{Methods}

The first patient harbouring G lamblia trophozoites in the gastric mucosa was observed in December 1988. All subsequent gastric biopsy specimens were routinely evaluated for the presence of this parasite, and a retrospective analysis was performed on all gastric biopsy specimens dating back to 1986 . Thus a total of 15023 specimens taken from 1986 to 1991 from 11095 patients were included in the current investigation.

When $G$ lamblia was morphologically identified, additional sections were cut from paraffin wax blocks and immunostained with a 1 in 10000 dilution of anti-Giardia polyclonal antiserum (Chemicon, Temecula, California, USA) and a standard avidin-biotin complexperoxidase technique (reagents from Dako, Glostrup, Denmark), as previously reported. ${ }^{6}$ All cases were also morphologically investigated for Helicobacter pylori infection. Histopathological changes in the gastric mucosa were recorded according to the Sydney system.

Nine patients found to harbour gastric $G$ lamblia on initial examination, had a repeat endoscopy before treatment; at that time both gastric and duodenal biopsy specimens were taken from each patient. In three of these cases Italy

Accepted for publication 6 May 1992 
part of the gastric samples were fixed in $2.5 \%$ glutaraldehyde in Millonig's phosphate buffer for four hours and processed for electron microscopic examination. Seven patients had a third control endoscopy one to two months after treatment.

Symptoms leading to endoscopy, other pertinent clinical data, and the effects of treatment (metronidazole $250 \mathrm{mg}$ three times a day for 10 days often associated with antacid drugs) in 24 patients who had been treated (patients seen after December 1988) were retrieved from the clinical records of each patient.

Twenty seven small bowel biopsy specimens, taken from patients investigated for malabsorption during the same period were also reviewed: 10 of these patients also had antral and fundic gastric biopsy specimens taken at the same time.

Finally, to evaluate the prevalence of $G$ lamblia in the population, we analysed duodenal biopsy specimens and cytological brushings of a consecutive series of 110 patients undergoing routine upper gastrointestinal tract endoscopic examination.

\section{Results}

IDENTIFICATION AND LOCALISATION OF

TROPHOZOITES

Forty one $(0.37 \%)$ of the 11085 patients under study harboured gastric $G$ lamblia infection. The trophozoites were identified at light microscopy as pear-shaped structures with two small paired nuclei, or as concave disks with thick outline (fig 1); thin flagella were occasionally seen. In all cases the immunocytochemical investigation provided confirmatory evidence for the identity of the trophozoites, which were strongly immunoreactive to the anti-Giardia antiserum (fig 2).

In the three cases submitted for electron microscopic examination the trophozoites,

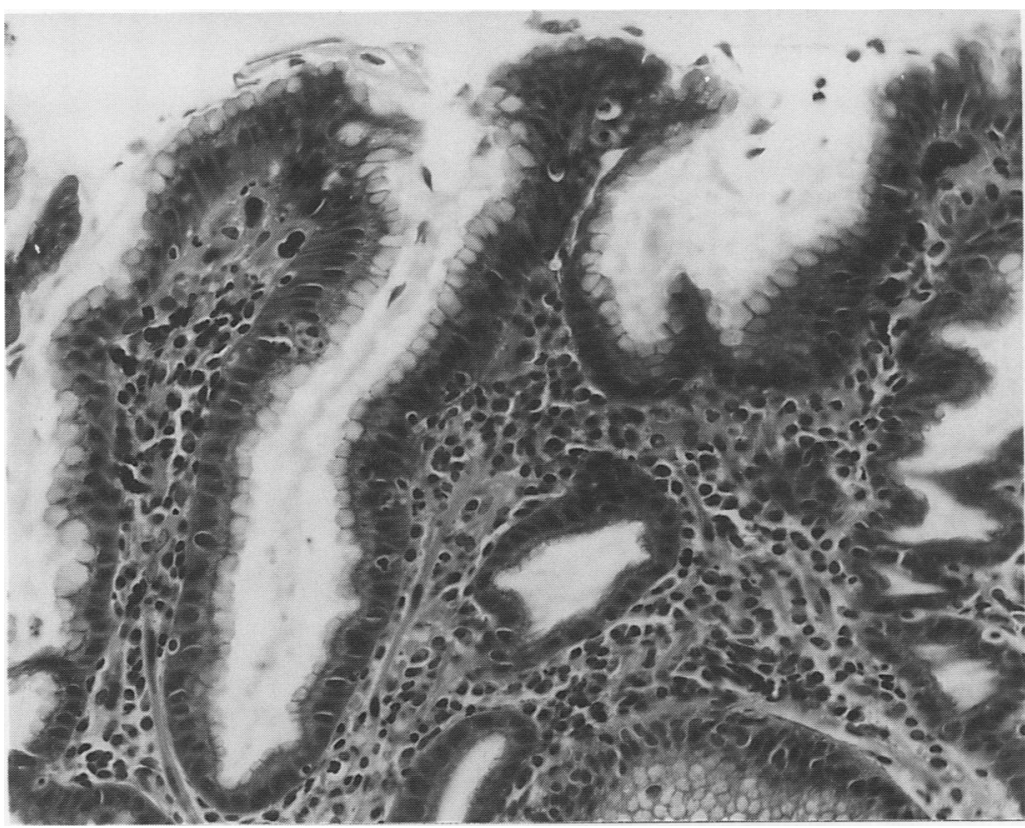

Figure 1 Light microscopic appearance of $G$ lamblia trophozoites overlying the superficial and foveolar epithelium of the gastric mucosa.

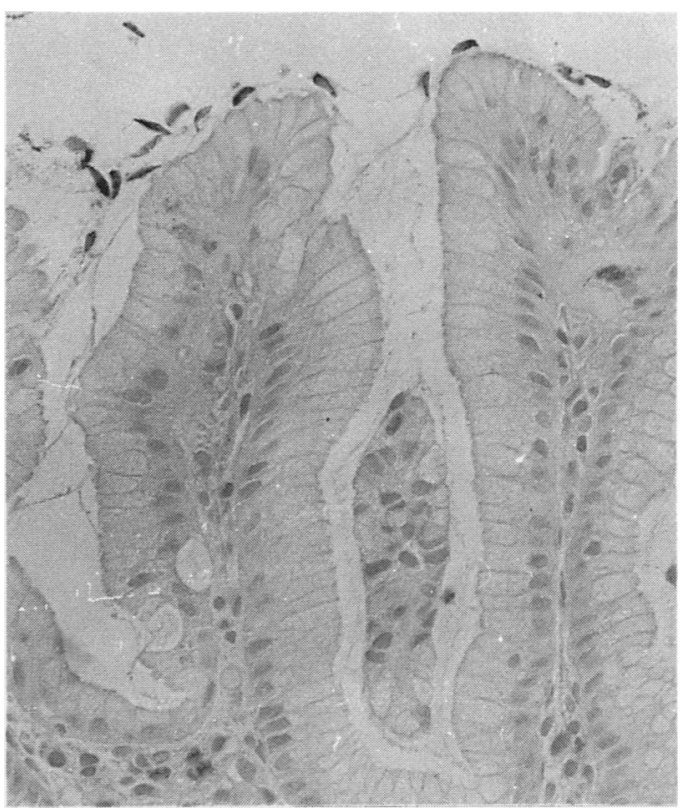

Figure 2 Trophozoites are immunoreactive to the anti-Gardia polyclonal antiserum.

measuring $12-15 \mu \mathrm{m}$ in maximum diameter, showed the typical ultrastructural features of $G$ lamblia, ${ }^{89}$ with a pair of ventral sucking disks and axostyles crossed by two parabasal bodies (fig 3). Four blepharoplasts and eight flagella were clearly evident in step sections of the same parasite.

Trophozoites were localised at the surface and at the base of foveolar pits, overlying the superficial and foveolar epithelium, and also in the intestinal metaplastic epithelium. Their number varied, ranging from five to more than 100 in each biopsy specimen. In specimens taken from gastric stumps, and including jejunal mucosa, $G$ lamblia was localised on both gastric and intestinal epithelia. G lamblia was never observed over fundic-type gastric mucosa.

CLINICOPATHOLOGICAL DATA

Dyspepsia, epigastric pain, and abdominal distension were the symptoms leading to endoscopic examination of the upper gastrointestinal tract in the 41 patients with gastric giardiasis. Only two patients had diarrhoea at the time of endoscopy. Four patients had recently travelled abroad (Mexico, Turkey, former Yugoslavia, Greece). None had detectable human immunodeficiency virus (HIV) serum antibodies; serological data available for 13 patients did not indicate any abnormality in serum immunoglobulins.

Cases of gastric giardiasis were diagnosed sporadically all year long, without any apparent seasonal exacerbation of the infection. Furthermore, there did not seem to be any clustering of the affected patients within specific regional areas.

The most relevant clinicopathological data of the 41 patients with gastric giardiasis are summarised in the table. All patients showed moderate to severe chronic atrophic gastritis with mild to severe activity, often associated with focal to extensive intestinal metaplasia. Concomitant $H$ pylori infection was identified 
Figure $3 A$ Semithin section of a plastic embedded gastric biopsy specimen showing $G$ lamblia colonisation of the gastric mucosa.

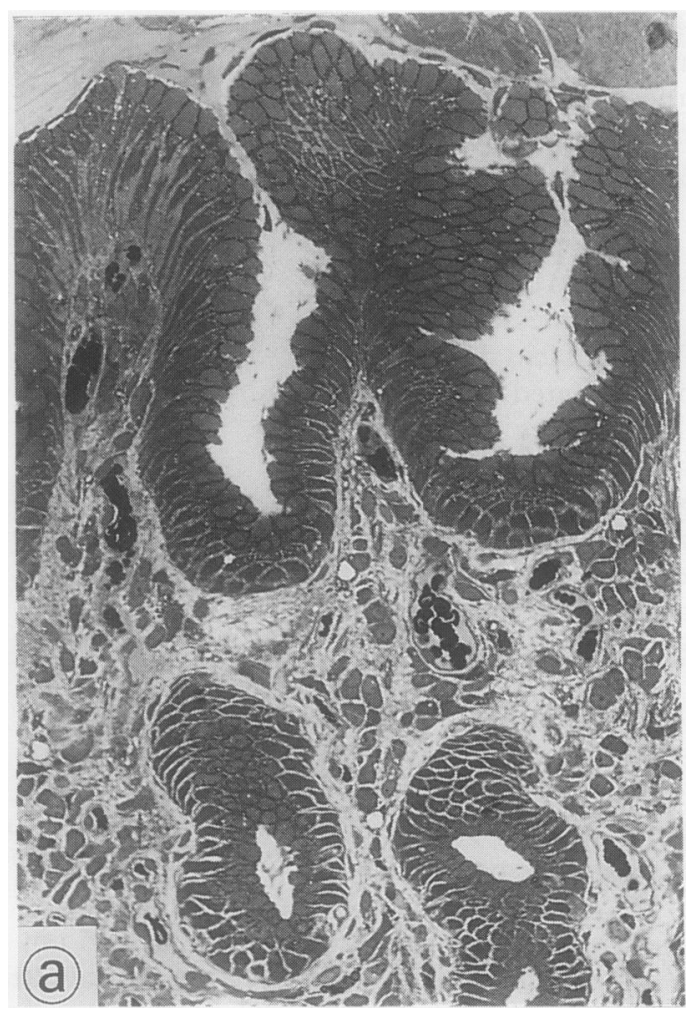

in 37 patients. Other associated gastric diseases included peptic ulcer $(n=2)$ and intestinaltype adenocarcinoma $(n=2)$. Eleven patients had previous partial gastrectomy (Billroth II) for peptic ulcer $(n=9)$ or gastric adenocarcinoma $(n=2)$. Five patients with previous histologically confirmed gastric peptic ulcer, not associated with $G$ lamblia infection showed gastric giardiasis in biopsy specimens taken 40
Relevant clinicopathological data of 41 patients with gastric giardiasis

\begin{tabular}{lc}
\hline Age range & $30-93$ (mean 60 years) \\
Male/female & $27 / 14$ \\
Chronic atrophic gastritis & $41 / 41$ \\
Intestinal metaplasia & $32 / 41$ \\
Helicobacter pylori infection & $37 / 41$ \\
Gastric ulcer & $2 / 41$ \\
Gastric adenocarcinoma & $2 / 41$ \\
Previous partial gastrectomy & $11 / 41$ \\
Previous antacid treatment & $5 / 41$ \\
Concomitant intestinal giardiasis & $9 / 9$ \\
Chronic infection (3-6 years' dura- & $8 / 8$ \\
tion) & \\
\hline
\end{tabular}

days later, after treatment with omeprazole $(\mathrm{n}=2)$, ranitidine $(\mathrm{n}=2)$, and nizatidine $(n=1)$. Trophozoites were identified both on the gastric and on the duodenal mucosa of the nine patients who repeated endoscopy and who also had gastric and duodenal biopsies performed before treatment. $G$ lamblia infection had been eradicated in the seven patients who repeated endoscopy after treatment. $H$ pylori infection persisted. Treatment resulted in at least partial relief of symptoms in ail 24 treated patients. Four patients with gastric giardiasis had concomitant colorectal $(n=2)$, pancreatic, and laryngeal carcinoma.

Previous gastric biopsy specimens from eight patients with newly diagnosed gastric giardiasis were available for review. In all these cases $G$ lamblia infection was already present (albeit originally unrecognised) in the previous specimens, dating back three to six years.

Twelve of the 27 patients evaluated for malabsorption were shown to harbour G lamblia infection of the duodenal and jejunal mucosa; five of them also had concomitant gastric biopsy specimens showing histolog-
Figure $3 B$ Ultrathin section of the same tissue sample as in figure $3 a$. $G$ lamblia trophozoite $(T)$ with ventral sucking $\operatorname{disk}(s)$.

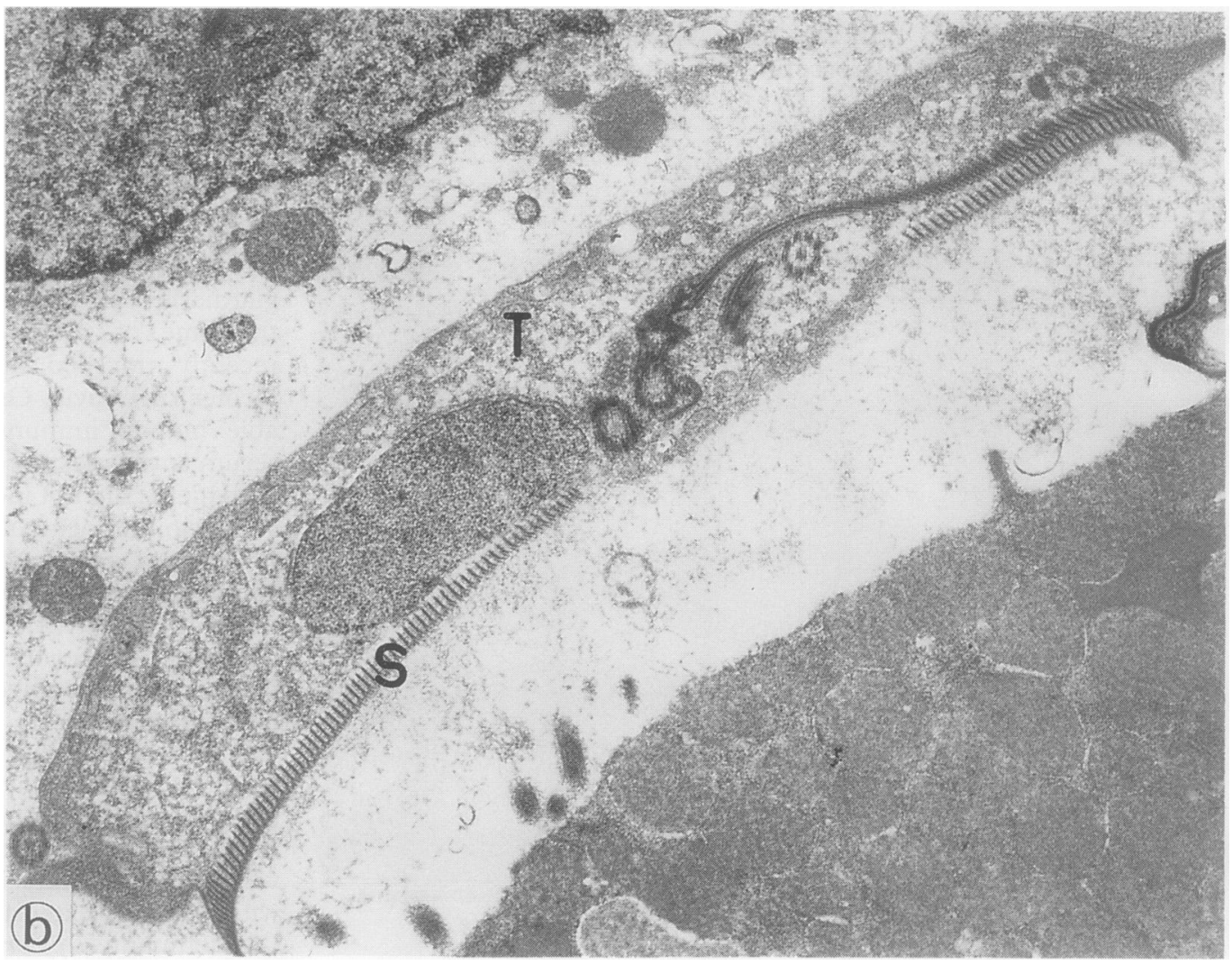


ically normal mucosa $(\mathrm{n}=2)$ or $H$ pylori associated chronic gastritis with mild atrophy $(n=3)$. Gastric giardiasis was absent in these patients.

Finally, only one of the 110 consecutive patients undergoing upper gastrointestinal tract endoscopy showed evidence of $G$ lamblia infection in the duodenal mucosa.

\section{Discussion}

To the best of our knowledge, this is the first report on the localisation of $G$ lamblia trophozoites to the gastric mucosa. The parasites have previously been identified in gastric lavage fluids, ${ }^{10}$ but contamination with duodenal juice of these specimens cannot be precluded. The current study is based on a population of almost 80000 inhabitants living in a restricted area of north eastern Italy, with a low prevalence of giardiasis (less than $1 \%$ of the patients undergoing upper gastrointestinal tract endoscopy, as documented in the present series). Therefore, although our results do not come from a multicentre investigation, the reported prevalence of $G$ lamblia trophozoites in $0.37 \%$ of gastric biopsy specimens may well be considered applicable to other areas with medium to high socioeconomic standards.

The small bowel mucosa is the expected area of colonisation of $G$ lamblia in infected patients; in this alkaline environment the organism can complete its life cycle and exert its pathogenic effects. ${ }^{11}$ A significantly decreased gastric acidity and modifications of the normal microenvironmental conditions of the gastric mucosa (becoming like those of the small bowel) may be essential prerequisites for $G$ lamblia colonisation of the stomach. Indeed, we were unable to find any gastric infection in patients with duodenal giardiasis and normal or slightly modified gastric mucosa. Conversely, we identified $G$ lamblia trophozoites only in the gastric biopsy specimens of patients with chronic atrophic gastritis, most often associated with $H$ pylor infection and showing intestinal metaplasia, and in gastric stumps. Both these conditions are known to reduce significantly or completely abolish gastric acidity. Furthermore, five patients showed gastric colonisation only after treatment with antacid secreting drugs for peptic ulcer.

Gastric giardiasis may be chronic: eight patients in our series had previous gastric biopsy specimens (dating back three to six years) which already harboured $G$ lamblia infection. At that time, however, we were unaware of the possible occurrence of gastric giardiasis and the presence of the trophozoites was therefore overlooked.

The pathogenic effects of $G$ lamblia on gastric mucosa are difficult to evaluate, especially because the histopathological changes we observed were probably attributable to concomitant $H$ pylori infection. Specific morphological changes due to $G$ lamblia colonisation, however, have not been identified even in the small bowel mucosa. ${ }^{12}$ Furthermore, $G$ lamblia infection was clinically unsuspected in all the $\mathbf{4 1}$ patients with gastric giardiasis. They mostly complained of dyspepsia, epigastric pain, or abdominal distension, although a recent study refuted $G$ lamblia as a cause of non-ulcer dyspepsia. In any case the above symptoms are not specific and may well be due to the underlying chronic atrophic gastritis and to $H$ pylori infection. ${ }^{13}$

Treatment with metronidazole and antacid drugs resulted in at least partial relief of the symptoms, but it was impossible to evaluate separately the effects of these medications. Metronidazole in the doses used, however, was ineffective at eradicating $H$ pylori infection.

Although the possible role of $G$ lamblia as a gastric pathogen remains to be clarified, we believe that a careful search for trophozoites should become part of the routine histopathological examination of gastric biopsy specimens, with particular care given to those showing chronic atrophic gastritis, and of gastric stumps. Because gastric giardiasis is invariably associated with intestinal giardiasis, the identification of $G$ lamblia in gastric biopsy specimens allows the infection to be diagnosed even if clinically unsuspected (symptoms related to $G$ lamblia infection are often atypical, as pointed out in recent studies, ${ }^{14}$ and diverse strains of this parasite may exert different pathogenic effects ${ }^{15}$ ) and for appropriate treatment to be given.

The demonstration of the actual capability of $G$ lamblia to colonise chronically the gastric mucosa might well stimulate further investigations aimed at better defining the interactions between man and this most common parasite.

1 WHO Expert Committee. Intestinal protozoan and helmintic infections. WHO Technical Report Series 666. Geneva: tic infections. WHO Technical Report
World Health Organization, 1981.

2 Chester AC, MacMurray FG, Restifo MD, Mann O. Giardiasis as a chronic disease. Dig Dis $\mathrm{Sc}$ 1985;30:215-8.

3 Green EL, Miles MA, Warhurst DC. Immunodiagnostic detection of Giardia antigen in faeces by a rapid visual enzyme-linked immunosorbent assay. Lancet 1985;ii: 691-3.

4 Butcher PD, Farthing MJG. DNA probes for the faecal diagnosis of Giardia lamblia infections in man. Biochem Soc Trans 1989;17:363-4.

5 Goldstein F, Thornton JJ, Szydlowski T. Biliary tract dysfunction in giardiasis. Am $\mathcal{F}$ Dig Dis 1978;23:559-60.

Doglioni C, Dell'Orto P, Coggi G, Iuzzolino P, Bontempini L, Viale G. Choroid plexus tumors. An immunocytochemical study with particular reference to the coexpression of intermediate filaments. $A m \quad \mathcal{F}$ Patho 987;127:519-29.

7 Misiewicz JJ, Tytgat GNJ, Goodwin CS, et al. The Sydney system: a new classification of gastritis. In: Working Party Report. Melbourne: Blackwell Scientific Publications, 1990:1-10.

8 Morecki R, Parker JG. Ultrastructural studies of the human Giardia lamblia and subjacent jejunal mucosa in a subject with steatorrheoea. Gastroenterology 1967;52:151-64.

9 Brookes SEH, Audretsch J, Muller CG, Sparke B. Electron microscopy of Giardia lamblia in human jejunal biopsies. f Med Microbiol 1970;3:196-9.

10 Koss LG. The gastrointestinal tract. In: Diagnostic cytology and its histopathological bases. Philadelphia: JB Lippincott, and its histopath

11 Ferguson A, Gillon J, Munro G. Pathology and pathogenesis of the intestinal mucosal damage in the giardiasis. In Meyer EA, ed. Giardiasis. Amsterdam: Elsevier, 1990:155-95.

12 Oberhuber G, Stolte M. Giardiasis: analysis of histological changes in biopsy specimens of 80 patients. $\mathcal{F}$ Clin Pathol 1990;43:641-3.

13 Carr MF, Ma J, Green PHR. Giardia lamblia in patients undergoing endoscopy: Lack of evidence for a role in non-ulcer dyspepsia. Gastroenterology 1988;95:972-4.

14 Gillon J. Clinical studies in adults presenting with giardiasis to a gastro-intestinal unit. Scot Med f 1985;30:89-95.

15 Nash TE, Herrington DA, Losonsky GA, Levine MM. Experimental human infections with Giardia lamblia. $\mathscr{J}$ Infect Dis 1987;156:974-84. 